\title{
Numerical study of mean-field approach capabilities for shape memory alloys matrix composites description
}

\author{
Y. Chemisky ${ }^{1, a}$, B. Piotrowski ${ }^{2}$, T. Ben Zineb ${ }^{2}$, and E. Patoor ${ }^{1}$ \\ ${ }^{1}$ LPMM, Metz University, Arts et Metiers ParisTech, CNRS, Ile du Saulcy 57045 Metz, France \\ 2 LEMTA, Nancy University, CNRS, 2 rue Jean Lamour, 54500 Vandoeuvre-les-Nancy, France
}

\begin{abstract}
With the development of SMA devices, new alloys are developed to enhance some specific properties like temperature hysteresis, plastic yield limit, change of transformation temperatures... Phase precipitation or inclusions addition are often use for these purposes. To predict the behavior of these heterogeneous materials and allow structures simulations, mean-field approaches like MoriTanaka homogenization scheme are convenient. But for phase-transformation strain mechanism, localization of deformation and, in a second step, saturation of transformation strain around inclusions have a significant impact on the global behavior. To check the capability of Mori-Tanaka scheme to predict the behavior of these heterogenous functional materials, a unit cell finite element analysis is considered as reference solution. One inclusion is embedded in the matrix, with respect to the fraction volume of both phases. Two situations are performed, both for NiTi-based materials. First represents the precipitation effect of Ni-rich elastic precipitates $\mathrm{Ni}_{4} \mathrm{Ti}_{3}$ for annealed Ni-rich materials. Second case considers elastoplastic $\mathrm{Nb}$ inclusions in a NiTi matrix, which induce a wide variation of reverse transformation temperatures. Limits of the homogenization approach chosen are studied in these two cases.
\end{abstract}

\section{Introduction}

Understanding effects of alloying, annealing and cold work on the martensitic transformation occuring in shape memory alloys will enhance functionnal properties of these materials. To consider the impact of these processing conditions, microscopic observations have to be coupled with models which link the evolution of microstructure and macroscopic behavior. Nowadays, several micromechanical models are developped, with some specificities. To model the influence of a few fraction of inclusions, Mori-Tanaka scheme [1] is often considered. The advantage is that an analytical solution is obtained with ellipsoidal-shaped inclusions. So this kind of model can be easily implemented in FEM codes.

For NiTi shape memory alloys, effect of $N i_{4} \mathrm{Ti}_{3}$ precipitates formation in Ni-rich $\mathrm{NiTi}$ materials when annealing is performed, or the addition of plastic inclusions of $\mathrm{Nb}$ have to be simulated. In these two cases, the inclusion volume fraction is always smaller or equal to $10 \%$, which is a suitable case for Mori-Tanaka scheme. Several works were done about the problem of elastic inclusions in an elastic-plastic matrix [2] [3] [4]. It appears that the incremental formulation delivers a stiffer response compared to experiments or numerical solutions. Some issues have been presented, but always with limitations. The aim of this study is to check, in case of specific SMA behavior by using an incremental (Hill's [5]) formulation for the homogenization

\footnotetext{
${ }^{a}$ e-mail: chemisky@univ-metz.fr
} 
scheme, the capability of predicting the correct macroscopic behavior. To consider the two cases of precipitation and alloying (addition of $\mathrm{Nb}$ ), the inclusion behavior is either elastic or elasticplastic. The matrix behavior is simulated using a specific thermodynamic macroscopic law developped by Chemisky et al. [6], and briefly described below. A FEM unit cell simulation model is considered as reference solution, in the same way of comparison made by Chaboche et al. in the case of an elastic inclusion embedded in an elastic-plastic matrix [4].

\section{Modeling}

\subsection{Shape memory alloy macroscopic law}

In shape memory alloys, several mechanisms induce observed macroscopic strain. They can be classified as follow:

- $E^{e}$ is the elastic strain;

- $E^{\alpha}$ is the strain induced by thermal expansion;

- $E^{T}$ is the inelastic strain due to martensitic transformation;

- $E^{\text {twin }}$ is the inelastic strain due to the accommodation of twins between martensite variants (in NiTi materials);

- $E^{P}$ is the plastic strain, neglected here;

It is assumed that thermoelastic parameters are the same in both phases. An additive decomposition of total strain is made in the framework of small perturbations:

$$
E_{i j}=E_{i j}^{e}+E_{i j}^{\alpha}+E_{i j}^{T}+E_{i j}^{t w i n}
$$

Transformation strain can be expressed as an average value in the RVE of local transformation strain. Local strain takes value described by the equivalent WLR and Bowles-Mackenzie theories [7] [8] which depends on formed variants. The macroscopic strain is defined by using two variables : the volume fraction of martensite $f$ and the mean transformation strain $\bar{\varepsilon}_{i j}^{T}$ :

$$
E_{i j}^{T}=\frac{1}{V} \int_{V} \varepsilon_{i j}^{T}(r) d V=f \bar{\varepsilon}_{i j}^{T}
$$

$E^{\text {twin }}$ is the macroscopic strain resulting from inelastic accommodation of twins inside martensite variants. A local strain is associated to this accommodation, according to observations made by [9] in NiTi samples. The resulting macroscopic strain is described by using a mean value of strain accommodation $\bar{\varepsilon}_{i j}^{t w i n}$ inside formed self-accommodated volume of martensite $f^{F A}$ :

$$
E_{i j}^{t w i n}=\frac{1}{V} \int_{V} \varepsilon_{i j}^{t w i n}(r) d V=f^{F A} \bar{\varepsilon}_{i j}^{t w i n}
$$

Three internal variables are used in the model:

- martensite volume fraction $f$;

- mean transformation strain in martensite volume $\bar{\varepsilon}_{i j}^{T}$;

- mean twins accommodation strain in twinned martensite volume $\bar{\varepsilon}_{i j}^{t w i n}$.

The formed self-accommodated martensite volume fraction $f^{F A}$ depends on the values of $f$ and $\bar{\varepsilon}_{i j}^{T}$ along the thermomechanical applied path. For example, a stress-free cooling leads to $f^{F A}=f$, and a superelastic path induce $f^{F A}=0$.

A thermodynamical potential is defined at the two-phased state. The Gibbs energy writes, assuming a decomposition of the elastic energy stored (interaction energy) and considering a linear variation of the entropy around an equilibrium temperature $T_{0}$ : 


$$
\begin{aligned}
\Delta G & =-\Delta T S^{A}+B\left(T-T_{0}\right) f-\frac{1}{2} \Sigma_{i j} S_{i j k l} \Sigma_{k l}-\Sigma_{i j} \alpha_{i j} \Delta T-\Sigma_{i j} f \bar{\varepsilon}_{i j}^{T} \\
& -\Sigma_{i j} f^{F A} \bar{\varepsilon}_{i j}^{t w i n}+\frac{1}{2} f H_{\varepsilon} \bar{\varepsilon}_{i j}^{T} \bar{\varepsilon}_{i j}^{T}+\frac{1}{2} H_{f} f^{2}+\frac{1}{2} f^{F A} H_{t w i n} \bar{\varepsilon}_{i j}^{t w i n} \bar{\varepsilon}_{i j}^{t w i n}
\end{aligned}
$$

Clausius-Duhem inequality writes, considering driving force variables $A_{k}$ linked to internal variables $V_{k}$ :

$$
-\frac{\partial \Delta G}{\partial \Sigma}: \dot{\Sigma}-\frac{\partial \Delta G}{\partial T} \cdot \dot{T}-\left\{A_{k}\right\} \cdot\left\{\dot{V}_{k}\right\}-\dot{\Sigma}: E-q_{i} \cdot \frac{T, i}{T} \geq 0
$$

Both transformation and orientation strain mechanisms are taking into account considering this formulation. Lagange multipliers are added to respect the physical limitations on martensite volume fraction $(0 \leqslant f \leqslant 1)$, and on mean transformation strain $\left(\bar{\varepsilon}_{e q}^{T} \leqslant \varepsilon_{M A X}^{T}\right)$. This last condition includes tension-compression assymetry and volume fraction of formed self-accommodated martensite [10]. Internal loops behavior is taking into account following the work of Peultier [11]. These assumptions lead to evolution laws for the three internal variables.

\subsection{Homogenization scheme considered}

An incremental Mori Tanaka scheme is considered, by using tangent operators for the twophases. By assuming that the strain rate on the boundary of inclusion is assumed to be equal to the strain rate in the matrix, then :

$$
\dot{\varepsilon}_{i j}^{I}=\dot{\varepsilon}_{i j}^{M}+T_{i j k l}\left(L_{k l m n}^{M}-L_{k l m n}^{I}\right) \dot{\varepsilon}_{m n}^{I}
$$

$L$ represent the mechanical tangent operators, $I$ stands for inclusion and $M$ for the matrix. $T$ is a fourth order interaction tensor derived from Eshelby inclusion theory. The global strain rate is assumed to be the sum between inclusion strain rate and matrix strain rate, each one being pondered by its volume fraction ( $f^{I}$ is the volume fraction of inclusions).

$$
\dot{E}_{i j}=f^{I} \dot{\varepsilon}_{i j}^{I}+\left(1-f^{I}\right) \dot{\varepsilon}_{i j}^{M}
$$

The concentration fourth order tensor is introduced.

$$
\dot{\varepsilon}_{m n}^{I}=A_{m n i j} \dot{E}_{i j}
$$

From equations 6 and 7 it comes:

$$
A_{m n i j}=\left[I_{i j m n}-\left(1-f^{I}\right) T_{i j k l}\left(L_{k l m n}^{M}-L_{k l m n}^{I}\right)\right]^{-1}
$$

The strain inside matrix is determined as:

$$
\dot{\varepsilon}_{i j}^{M}=\frac{1}{1-f^{I}}\left(\left(I_{i j m n}-f^{I} A_{i j m n}\right) \dot{E}_{m n}\right)
$$

The strain in each phase can derived from macroscopic strain. So, by taking into account the constitutive equations for inclusion and matrix, the effective constitutive law is derived as follow:

with:

$$
\dot{\Sigma}_{i j}=L_{i j m n}^{e f f} \dot{E}_{m n}-M_{i j}^{e f f} \dot{T}
$$

$$
\begin{aligned}
& L_{i j m n}^{e f f}=f^{I}\left(L_{i j k l}^{I}-L_{i j k l}^{M}\right) A_{k l m n}+L_{i j m n}^{M} \\
& M_{i j}^{e f f}=f^{I} C_{i j k l}^{I} \alpha_{k l}^{I}+\left(1-f^{I}\right) M_{i j}^{M}
\end{aligned}
$$


In these scheme $L_{i j k l}^{I}$ correspond to the elastic or elastic-plastic behavior of the inclusion, and $L_{i j k l}^{M}$ is determined by solving the evolutions laws equation system related to the SMA model. $M_{i j}$ represent the tangent thermal operator.

\section{Comparison between homogenization scheme and numerical solution}

Numerical unit cell simulations are used as reference solution to test the capabilities of MoriTanaka scheme. Inclusions repartition is assumed statistically homogeneous, with perfect interfaces between inclusion and matrix. A cubic unit cell containing one spherical inclusion is then defined. Symmetries allow to simulate only $\frac{1}{8}$ of the unit cell. Simulations are performed using Abaqus FEA package. Uniaxial isothermal tensile loading paths are performed at various temperatures, with displacement boudary conditions, until a limit mean global strain (6\%).

The SMA matrix behavior model is implemented through a UMAT (for User MATerial) subroutine. Material parameters for the SMA model are resumed in table 1.

Table 1. Material parameters for NiTi matrix

\begin{tabular}{clllll}
\hline$E(M P a)$ & $\nu$ & $\alpha\left({ }^{\circ} \mathrm{C}\right)^{-1}$ & $\varepsilon_{\text {tracmax }}^{T}$ & $\varepsilon_{\text {tractmax }}^{T F A}$ & $\varepsilon_{\text {compmax }}^{T}$ \\
70000 & 0.3 & 0 & 0.05 & 0.05 & 0.04 \\
\hline$b_{d}\left(M P a /{ }^{\circ} \mathrm{C}\right)$ & $b_{r}\left(M P a /{ }^{\circ} \mathrm{C}\right)$ & $M_{S}\left({ }^{\circ} \mathrm{C}\right)$ & $A_{f}\left({ }^{\circ} \mathrm{C}\right)$ & $r_{f}$ & \\
5 & 5 & 10 & 40 & 0.25 & \\
\hline$F_{\varepsilon}(M P a)$ & $H_{f}(M P a)$ & $H_{\varepsilon}(M P a)$ & $H_{t w i n}(M P a)$ & $H_{s}(M P a)$ & \\
200 & 2 & 1200 & 30000 & 30 & \\
\hline
\end{tabular}

For elastic inclusions case, three tests are performed for $-20,30$ and $60^{\circ} \mathrm{C}$ temperatures, which are under $M_{s}$, between $M_{s}$ and $A_{f}$ or above $A_{f}$. This lead to a wide range of behavior for the SMA matrix, because orientation or/and transformation strain mechanisms are activated. Elastic behavior of inclusions are chosen with respect $N i_{4} T i_{3}$ elastic constants calculated from first principle by Wagner et al. [12]. Three volume fractions are considered, respectively 2, 5 and $10 \%$. A uniaxial loading path is applied, until a maximal strain of $6 \%$ followed by an unloading to $0 \%$ strain. The comparison between Mori-Tanaka scheme predictions and Unit Cell simulations are presented figure $1 \mathrm{a}), \mathrm{b}$ ) and c). One can observe several differences between these two models. Mori-Tanaka scheme predicts a stiffer pseudo-hardening at the very beginning of non-elastic strain mechanism activation, for both orientation behavior $\left(-20^{\circ} \mathrm{C}\right)$, mixed transformation/orientation $\left(30^{\circ} \mathrm{C}\right)$ or transformation behavior $\left(60^{\circ} \mathrm{C}\right)$. This pseudo-hardening is strongly dependent on the volume fraction of inclusions. Before $3 \%$ deformation value, the Unit Cell response is weakly dependent on volume fraction of inclusions. On the other hand, after a deformation of $3 \%$, the response is became stiffer, and the stress value at $6 \%$ is in the same order of Mori-Tanaka response. These differences highlight the influence of transformation and/or orientation heterogeneity in a unit Cell. For example, figure 2 presents the martensite volume fraction $\left(\right.$ at $60^{\circ} \mathrm{C}$ ) and the equivalent mean transformation strain $\left(\right.$ at $\left.-20^{\circ} \mathrm{C}\right)$. These two variables are characteristics of the macroscopic transformation strain in the two selected cases. Similar fields are observed, which emphasize the strong effect of precipitate geometry and boundary conditions.

With respect to microscopics observations made by [13] on commercial $N i_{47} T i_{44} N b_{9}$ materials, volume fraction of Nb-rich precipitates is approximatively $10 \%$. Three simulations are performed, for respectively $-20,30$ and $60^{\circ} \mathrm{C}$, considering elastic-plastic inclusions, with a Young modulus value of $52 \mathrm{GPa}$ and a Poisson's ratio of 0.3 , to respect Niobium behavior. Plastic strain evolution is detailled in table 2, coming from experiments performed by NematNasser and Guo on pure Niobum [14].

The comparison between Mori-Tanaka scheme predictions and unit cell simulations are presented figure $1 \mathrm{~d}$ ). Discrepancies observed when saturation of transformation or orientation 

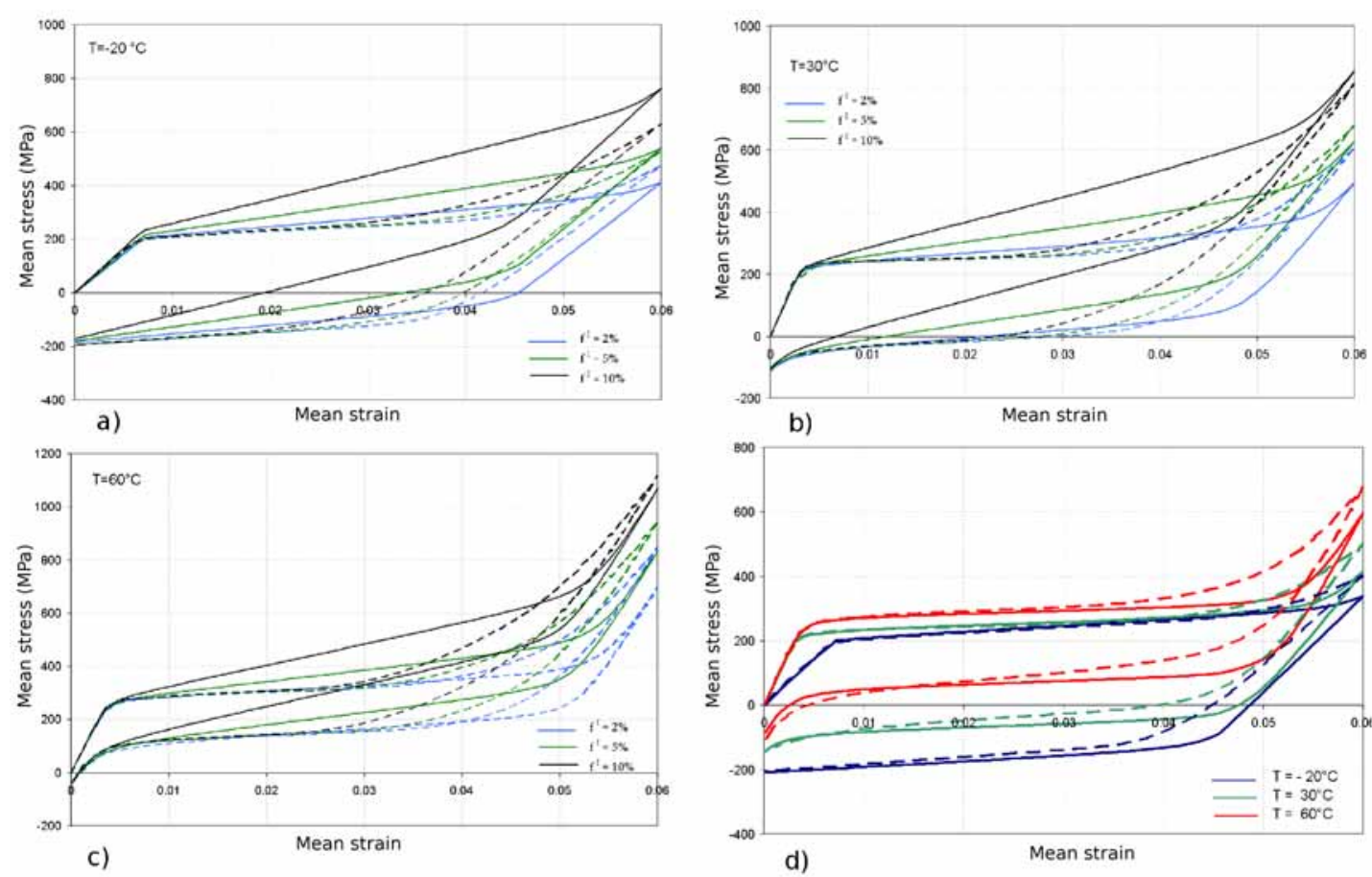

Fig. 1. a) , b), c) Macroscopic behavior with elastic inclusions at various temperatures. Plain lines : Mori-Tanaka response, dashed lines : Unit Cell simulations d) Macroscopic behavior with elasticplastic inclusions at various temperatures. Plain lines : Mori-Tanaka response, dashed lines : Unit Cell simulations

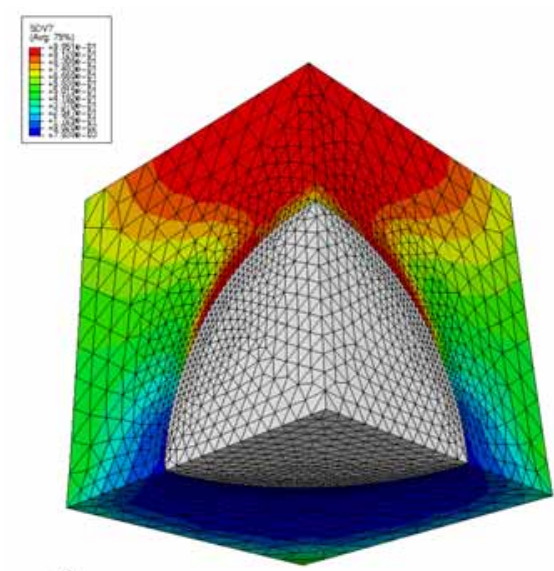

a)

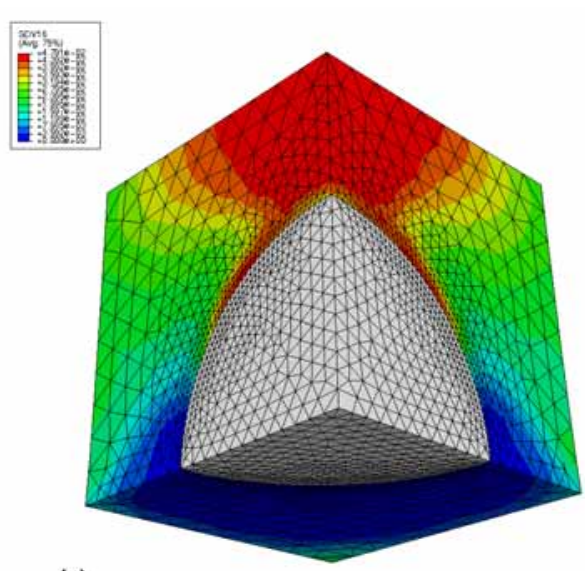

b)

Fig. 2. a) Evolution of martensite volume fraction, $\left(60^{\circ} \mathrm{C}, \varepsilon=3 \%\right)$ b) Evolution of mean transformation strain, $\left(-20^{\circ} \mathrm{C}, \varepsilon=3 \%\right)$. Inclusion volume fraction in these two cases : $10 \%$

occurs (up to $4 \%$ strain). Mori-Tanaka scheme underestimate the overall response. But when the loading path is reversed, the two models responses are again very close. 
Table 2. Plastic deformation evolution

\begin{tabular}{cllllll}
\hline$\varepsilon^{p}$ & 0 & 0.0009 & 0.0039 & 0.0113 & 0.051 & 0.154 \\
$\sigma$ & 90 & 120 & 150 & 180 & 240 & 300 \\
\hline
\end{tabular}

\section{Discussion and Conclusions}

Comparisons between Mori-Tanaka and a unit cell simulation lead to several conclusions. For elastic inclusions, the MT model is too stiff when the transformation and/or orientation mechanism occur, which lead to a weak tangent stiffness for the matrix. But when saturation is reached, the overall responses are more closer. Moreover, in the case of orientation, the MT scheme response underestimate the unit cell simulation. Main difference with the case of elasticplastic materials is the saturation of transformation strain, which lead again to an elastic behavior (as long as stress are not to high).

Some studies done in plasticity have shown the same overestimation of the overall behavior when plastic strains occur in the matrix [4] [3]. One issue is the isotropisation of the Green strain tensor, which reduces the effective modulus of the composite [4]. But the physical meaning of this approximation is not clear. Another way is to consider a secant modulus, in the same way of Zaoui and Berveiller model for self-consistent scheme adapted to plasticity [15]. In this case, only monotonous and proportionnal loading can be simulated, which reduce the model capabilities (especially for structure computation). One prospect of this work is to compare several cases of homogenizations, including these features.

In the case of elastic-plastic inclusions, the MT predictions are very close compared to the unit cell simulations, except when saturation occurs. The homogenization method chosen is then adapted to model the effect of Niobium in NiTiNb materials. The principal purpose is to capture, with this model, the effect of martensite stabilization, linked with plastic strains generated in $\mathrm{Nb}$ inclusions ${ }^{1}$.

\section{References}

1. T. Mori and K. Tanaka, Acta Metallurgica, 21, (1973) 571-574.

2. G. Dvorak and Y. Benveniste, Proc. Roy. Soc. London Ser., A 437 (1992), 291-310

3. I. Doghri and A. Ouaar, IJSS, 40 (2003), 1681-1712

4. J.L. Chaboche, P. Kanouté and A. Roos, Int. J. of Plasticity 21, (2005) 1409-1434

5. R. Hill, J. Mech. Phys. Solids, 13 (1965), 89-101

6. Y.Chemisky, A. Duval, B. Piotrowski, T. Ben Zineb, V. Tahiri and E. Patoor, Smart Materials and Structures, accepted (2009)

7. M. Weschler, D. Lieberman and T. Read, Trans. AIME 197, (1953) 1503

8. J. Bowles and J. Mackenzie, Acta Metall. 2, (1954) 129-137

9. Y. Liu, Z. Xie, J. Van Humbeeck and L. Delaey, Scripta Materialia 41, (1999) 1273-1281

10. Y. Chemisky, Phd Thesis - Université de Metz (2009)

11. B. Peultier, T. Ben Zineb and E. Patoor, Mat. Science \& Eng. A, 481-482, (2008) 384-388

12. M.F.X. Wagner and W. Windl, Scripta Materialia, 60 (2009), 207-210

13. B. Piotrowski, A. Duval, T. Ben Zineb, E. Patoor, A. Eberhardt, F. Pauchet, J-L. Garcia, Proceedings of ICOMAT 2008 - TMS publishers, 2008

14. S. Nemat-Nasser and W. Guo., Mat. Science \& Eng. A, 284 (2000), 202-210

15. M. Berveiller, A. Zaoui, J. Mech. Phys. Solids, 26 (1979), 325-344

\footnotetext{
${ }^{1}$ B. Piotrowski, Phd Thesis, in progress
} 